\title{
CHANGES IN RESTING SALIVARY TESTOSTERONE, CORTISOL AND INTERLEUKIN-6 AS BIOMARKERS OF OVERTRAINING
}

\author{
Travis Anderson, Simon Haake, Amy R. Lane, Anthony C. Hackney \\ University of North Carolina, Chapel Hill, NC, USA
}

\begin{abstract}
Background. Overtraining (OVT) is a concern for many athletes. Immunological (increased interleukin-6 [IL-6]) and hormonal (increased cortisol [C], decreased free testosterone [fT]) biomarkers have been analyzed during training to detect OVT development.

Methods. This study determined if resting levels of salivary IL-6, T, and C change during a pre-season resistance training (RT) program in 20 Division I American football players (mean \pm SD: age $=19.1 \pm 1.1$ years; height $=$ $185.4 \pm 6.7 \mathrm{~cm}$; mass $=102.0 \pm 22.2 \mathrm{~kg}$; body fat $=14.7 \pm 7.6 \%)$. $1 \mathrm{RM}$ squat, bench press and Olympic-style clean, IL-6, C and T were assessed at baseline (WK1), week 4 (WK4), week 6 (WK6) along with psychological status (PS) to determine affective state.

Results. 1RM (bench press: $121.6 \pm 36.3 \mathrm{~kg}$ vs. $127.4 \pm 35.9 \mathrm{~kg}$, squat: $187.2 \pm 30.2 \mathrm{~kg}, 190.9 \pm 28.1 \mathrm{~kg}$, clean: $116.8 \pm 14.6 \mathrm{~kg}$, vs. $119.2 \pm 14.5 \mathrm{~kg})$, IL-6 $(1.42 \pm 1.77 \mathrm{pg} / \mathrm{mL}$ vs. $5.60 \pm 12.57 \mathrm{pg} / \mathrm{mL})$ and C $(2.57 \pm$ $2.46 \mathrm{nmol} / \mathrm{L}$ vs. $5.33 \pm 4.94)$ increased significantly from WK1 to WK6 $(p<.05)$, fT decreased significantly $(417.44 \pm 83.63 \mathrm{pmol} / \mathrm{L}$ vs. $341.10 \pm 87.79 \mathrm{pmol} / \mathrm{L})$ from WK1 to WK6 $(p<.05)$. PS was minimally affected during the study. Significant biomarker changes were detected, but no OVT was induced (i.e. performance improved).

Conclusion. Therefore, directional changes in these biomarkers may not be sufficiently reflective of OVT in RT programs.
\end{abstract}

Keywords: stress, hormones, biomarkers, anabolic-catabolic.

\section{INTRODUCTION}

$\mathrm{E}$ lite level strength and conditioning coaches are tasked with optimizing player performance. To achieve these goals, strength coaches often train athletes throughout the year at, or near, their maximal physical capacity using a process called overreaching. In the overreaching process, one or more training factors (e.g. modality, duration, intensity, volume) are increased beyond what the athlete is typically accustomed to in order to elicit a supercompensatory response (i.e. overload principle) (Meeusen et al., 2006). When overreaching training is excessive however, it can lead to a state known as "overtraining," characterized by a rapid deterioration in performance that does not respond to a rest or regeneration period (Lehmann, Foster, \& Keul, 1993). An athlete who is overtrained may display a myriad of physiological and psychological symptoms including chronic fatigue, decreased performance, depression, apathy towards training, and sleeplessness. Research findings suggest these symptoms may be largely attributed to a compromise in the immunological and neuroendocrine systems (Robson, 2003).

Intense or prolonged bouts of exercise can lead to the production and subsequent elevation of pro-inflammatory cytokines. These cytokines, such as interleukin- $1 \alpha$, interleukin-1 $\beta$, and interleukin-6 (IL-6) are part of an acute proinflammatory immune response to physiological 
stressors. During periods of overreaching proinflammatory cytokines may reach chronically elevated levels. Dr. Lucille Smith developed the 'Cytokine Hypothesis' to explain overtraining in athletes, specifically proposing that excessive production and/or heightened sensitivity in tissues to the specific cytokine IL-6 is the principle factor leading to the physiological changes in the overtrained state (Robson, 2003; Smith, 2000).

Research suggests that the neuro-endocrine system is also a valuable parameter to analyze when determining whether an athlete is overtrained (Hackney, 2006). During intense-prolonged exercise, the neuro-endocrine system releases hormones that moderate the metabolic response to exercise. These hormones also play an important role in the functioning of the immune system during exercise. Cortisol, the major human glucocorticoid hormone released in response to physical and psychological stress, is a strong effector factor of the immune response (Hackney \& Waltz, 2013). Additionally, it is well documented that in response to exercise training stress (i.e. if excessive) basal testosterone levels will become suppressed in men, perhaps due to the inhibitory actions of cortisol (Urhausen, Gabriel, \& Kindermann, 1995). With the above in mind, the purpose of this study was to evaluate select immunological and hormonal biomarker (salivary cortisol, testosterone, IL6) responses of men in a six-week intensive preseason training program to examine for signs of overtraining.

\section{METHODS}

Subjects were twenty moderate to highly strength-trained healthy male subjects (mean $\pm \mathrm{SD}$ : age $=19.1 \pm 1.1$ years; height $=185.4 \pm 6.7 \mathrm{~cm}$; mass $=102.0 \pm 22.2 \mathrm{~kg}$; body fat $=14.7 \pm 7.6 \%$ ) recruited from a Division I collegiate American Football team. All subjects were upper-classmen and constituted a variety of non-lineman positions. All signed a written informed consent in compliance with the Declaration of Helsinki. For eligibility subjects must have had full participation in team training activities for a minimum of 3 days a week for 3 months prior to the study and have had a two year history of resistance training consisting of at least one training session per week.

Each subject was assessed at six separate sessions. Anthropometric data (age, height, mass, body fat percentage) were collected at the first session. Body fat percentage was determined through triplicate skinfold measurements at select sites (abdomen, chest, and thigh) using skinfold calipers (Skyndex, Fayetteville, AR) and body fat percentage was calculated using the JacksonPollock equation (Golding, 2000). Salivary samples were collected at Week 1 (baseline), Week 4 and Week 6 while psychological status responses and body mass were collected weekly. The modified version of the Recovery-Stress Questionnaire for Athletes (REST-Q) was used to monitor psychological status over the course of the study, administered to the subjects on the morning of saliva sample collection.

Prior to collection of saliva samples, subjects were first asked to rinse their mouths with water, spit to eliminate particles, and then allow saliva to accumulate; samples were then collected via passive drool sampling technique. The time of day (afternoon; 15:00-17:00 H) for all salivary collections remained relatively consistent over the course of the study for each subject ( \pm 15 minutes). Samples were stored on ice until transported to a storage freezer $\left(-80^{\circ} \mathrm{C}\right)$ until later analysis.

Saliva samples were assessed for IL-6, free testosterone (fT) and cortisol (C) concentrations. Stored saliva samples were allowed to thaw and were then centrifuged at $3000 \mathrm{x} g$ at 4 degrees $\mathrm{C}$ to remove any particulate matter. The resulting supernated saliva specimens were assayed for cortisol, IL-6, and fT using high sensitivity enzyme immunoassays (Salimetrics, State College, PA, USA).

Training load, intensity, and volume were controlled by strength and conditioning staff and monitored by the principle investigator to ensure progressions remained within $+10 \%$ of baseline load. Muscle strength was assessed pre- and posttraining using 1 repetition maximum (1RM) for bench press, back squat and Olympic-style clean. Each training session consisted of a 10-15 minute warm-up comprised of three separate stations (core/ abdominal exercise, dynamic/static stretching and shoulder exercises), each lasting 3-5 minutes. Post warm-up, training consisted of whole body and body part isolated resistance training exercises including back squat, bench press, power clean, hang clean, incline bench press, lunges, and several assistance exercises (Table 1, all exercises). Each training session began with one of the primary exercises, progressing to near-maximum intensity. Supplementary exercises were completed after completion of the main lifts. Total session length was between $45-60$ minutes. 


\begin{tabular}{|c|c|c|c|c|c|}
\hline \multirow{2}{*}{$\begin{array}{l}\text { Table 1. Exercises used } \\
\text { throughout the 6-week } \\
\text { training period }\end{array}$} & Primary & Lower Body & \multicolumn{2}{|c|}{ Upper Body } & Compound \\
\hline & $\begin{array}{l}\text { Bench Press } \\
\text { Back Squat } \\
\text { Power Clean }\end{array}$ & $\begin{array}{l}\text { Front Squat } \\
\text { BB Lunges } \\
\text { RDL } \\
\text { Calf Raises } \\
\text { Deadlift }\end{array}$ & $\begin{array}{l}\text { DB Overhead Press } \\
\text { Push Ups } \\
\text { Incline Bench Press } \\
\text { Incline DB Press } \\
\text { Front Press } \\
\text { Close-Grip Bench P }\end{array}$ & $\begin{array}{l}\text { DB Curl } \\
\text { BB Curl } \\
\text { BB Row } \\
\text { Pull Ups } \\
\text { BB Shrugs }\end{array}$ & Hang Clean \\
\hline
\end{tabular}

Training volume increased gradually over the course of the study through manipulation of total sets completed per exercise and an increase in the number of exercises completed. Relative intensity (training stimulus as percentage of maximum capability), total training load, and training load for the primary exercises were catalogued to ensure an appropriate training stimulus was being applied.

Beginning on the second week of training conditioning runs were completed twice a week on Tuesdays and Thursdays. These conditioning sessions were executed immediately after the completion of the resistance training session and consisted of 100 or 300 -meter runs at a brisk predetermined pace. Each week, running volume was increased to create a higher physiological demand on the athlete. On the fifth and sixth weeks of training, the 300-meter runs were replaced with a speed/agility circuit lasting 45 minutes. The circuit consisted of 4 separate drills completed for a total of 8 minutes, with 2 minutes rest between drills.

Statistical Analysis. This quasi-experimental study was powered based upon anticipated biomarker responses, for a two-sided test with an effect size of $\geq 0.50$ and a power $(\beta)$ of 0.80 . The sample size required to show statistical significance with these parameters was calculated to range from 12 to18 subjects (Cohen, 1992).

Separate one way within subjects repeated measures ANOVA were used to analyze IL-6, fT, $\mathrm{C}$, body mass, and the REST-Q categories over measurement time. Tukey post-hoc tests were used to determine significant mean differences between levels within each ANOVA. Separate paired samples t-tests were used to determine changes in muscular strength assessments. Significance for all statistical tests was set a priori at $\alpha \leq 0.05$.

\section{RESULTS}

Weekly body mass $(\mathrm{kg})$ of subjects is displayed in Table 2 and increased significantly by the conclusion of the study.

Each of the subjects had completed 1RM on all major lifts used in the study (bench press, back squat, and power clean) prior to involvement and hence were familiar with the procedures performed per National Strength and Conditioning Association guidelines (Fry \& Kraemer, 1991). 1RM results during the six-week study period were catalogued. Pre- and post-training 1RM results are displayed in Table 3.

Table 2 . Weekly body mass (mean $\pm S \boldsymbol{D})$

\begin{tabular}{|c|c|c|c|c|c|c|}
\hline & Pre & Week 2 & Week 3 & Week 4 & Week 5 & Week 6 \\
\hline Body Weight (kg) & $102.0 \pm 22.2$ & $102.5 \pm 22.3^{*}$ & $102.8 \pm 22.1^{*}$ & $103.0 \pm 22.4^{*}$ & $103.3 \pm 22.4^{*}$ & $102.8 \pm 22.2$ \\
\hline
\end{tabular}

Notes. *Indicates significant change from baseline. $\gamma$ Beginning of study (week 1) $(p<.05)$.

Table 3. Performance responses of main resistance exercise lifts (mean \pm SD)

Note. *Significant differences from respective prestudy value $(p<.05)$.

\begin{tabular}{|c|c|c|c|}
\hline \multicolumn{1}{|c|}{ Lift } & Pre-Study Max (kg) & Post-Study Max (kg) & Change (\%) \\
\hline Bench Press & $121.6 \pm 36.3$ & $127.4 \pm 35.9^{*}$ & $+4.8 \pm 4.2$ \\
\hline Back Squat & $187.2 \pm 30.2$ & $190.9 \pm 28.1^{*}$ & $+2.0 \pm 3.1$ \\
\hline Power Clean & $116.8 \pm 14.6$ & $119.2 \pm 14.5^{*}$ & $+2.1 \pm 3.3$ \\
\hline
\end{tabular}




\begin{tabular}{|l|c|c|c|}
\hline \multicolumn{1}{|c|}{ Hormone (units) } & Pre $^{\text {y }}$ & Week 4 & Week 6 \\
\hline Cortisol $(\mathrm{nmol} / \mathrm{L})$ & $2.57 \pm 2.46$ & $3.67 \pm 3.42$ & $5.33 \pm 4.94^{*}$ \\
\hline IL-6 $(\mathrm{pg} / \mathrm{mL})$ & $1.42 \pm 1.77$ & $4.19 \pm 8.27$ & $5.60 \pm 12.57^{*}$ \\
\hline Free Testosterone $(\mathrm{pmol} / \mathrm{L})$ & $417.44 \pm 83.63$ & $456.00 \pm 100.98$ & $341.10 \pm 87.79 *$ \\
\hline
\end{tabular}

Table 4. Salivary biomarker concentrations (SI units) mean $\pm S D$ )

Notes. *Significant difference from Week 1 trial $(p<$ .05). $\gamma$ Beginning of week 1 .

\begin{tabular}{|l|c|c|c|c|c|c|}
\hline \multicolumn{1}{|c|}{$\begin{array}{c}\text { Affective } \\
\text { Category }\end{array}$} & Pre-Study & Week 2 & Week 3 & Week 4 & Week 5 & Week 6 \\
\hline Anger & $1.56 \pm 1.2$ & $1.38 \pm 1.5$ & $1.05 \pm 1.2^{*}$ & $1.36 \pm 1.7$ & $0.83 \pm 1.3^{*}$ & $1.15 \pm 1.4^{*}$ \\
\hline Depression & $1.67 \pm 1.6$ & $1.81 \pm 1.4$ & $1.32 \pm 1.3^{*}$ & $1.46 \pm 1.6$ & $1.62 \pm 1.8$ & $1.83 \pm 1.9$ \\
\hline Fatigue & $1.85 \pm 1.3$ & $1.91 \pm 1.6$ & $1.46 \pm 1.4^{*}$ & $1.54 \pm 1.5$ & $1.44 \pm 1.4^{*}$ & $1.56 \pm 1.4$ \\
\hline $\begin{array}{l}\text { Vigor } \\
\text { (Motivation) }\end{array}$ & $1.43 \pm 1.4$ & $\begin{array}{c}1.10 \pm \\
1.2^{*}\end{array}$ & $1.32 \pm 1.5$ & $1.23 \pm 1.5$ & $1.06 \pm 1.3$ & $0.92 \pm 1.1^{*}$ \\
\hline
\end{tabular}

Table 5. REST-Q score by affective category $($ mean $\pm S D)$

Notes. *Significant difference from respective prestudy (Week 1) baseline measure $(p<.05)$. Scale range: 1 (Low) -5 (High).

The mean $( \pm S D)$ resting salivary C, IL-6 and fT responses over the six-week study period are displayed in Table 4. C trended upward throughout the study. The increase from Week 1 to Week 4 was not significant $(p=.236)$. However, the difference between Week 1 and Week 6 was significant, showing an increase of approximately $110 \%(p=.004)$.

There were no significant differences between means for resting IL-6 over the course of the study $(p=.170)$. However, due to the large variability in the responses, the data was transformed (log base 10) and re-analyzed according to literature recommendations (Hackney \& Viru, 2008). This analysis showed a significant increase in IL-6 values from Week 1 to Week 6 , an increase of approximately $300 \%(p=.001)$.

fT did not change significantly from week 1 to week 4 ( $p=.411)$. There was, however, a significant reduction from week 1 to week 6 , a decrease of approximately $20 \%(p=.007)$.

Scores for each affective category on the REST-Q are displayed in Table 5. All affective categories scores (anger, depression, fatigue and vigor) were significantly reduced from baseline at various points during the study (see Table 5 for specific points of significance; N.B., increased scores meant greater amounts of affective status, except for vigor where increased scores meant decreased status).

\section{DISCUSSION}

Our aim was to investigate the combined responses of C, IL-6 and fT of American football athletes during an intensive pre-season training regimen. It was hypothesized that significant resting, basal increases in $\mathrm{C}$ and IL-6, and a decrease in fT would occur; and, potentially impair weight lifting performance due to increased sensations of fatigue, illness, and stress developing in accordance with the "Cytokine Hypothesis" of overtraining. Athletes who are overtrained have a variety of specific physiological and psychological symptoms - weight loss, performance decline, increased fatigue-depression, and reduced vigormotivation (Hackney, 2006; Smith, 2000; Urhausen et al., 1995). By the end of the 6-week training period in this study there were significant increases in each of the three 1RM exercises (performance improved), body weight increased and no adverse psychological changes occurred; in fact, slight positive affective changes developed. Based upon accepted criteria, the subjects in this study were not symptomatic of overtraining. Yet, the biomarker responses (decreased fT, increased C and IL-6) observed are indicative of the theoretical changes supposedly reflective of an overtrained state as has been proposed in the literature by several research groups (Hackney, 2006; Kraemer \& Ratamess, 2005; Robson, 2003; Smith, 2000; Urhausen et al., 1995).

Notably all the subjects were experienced weight lifters who had strength performances commensurate with elite American football athletes (Fry \& Kraemer, 1991; Hoffman \& Kang, 2003; Ware, Clemens, Mayhew, \& Johnston, 1995). Also, they were supervised in their training by both a qualified strength coach and research team 
member who designed and executed their strengthconditioning program utilizing a scientific, progressive overload methodology (Burke et al., 2001; Hoffman \& Kang, 2003). This program was intended to improve the athlete's performance, as well as subject them to a rigorous regimen associated with development of overtraining (Fry \& Kraemer, 1991). It is also critical to note the resting biomarker levels observed prior to the exercise intervention reflect valid resting levels, based on reference ranges provided by the assay manufacturer (Salimetrics, USA) as well as reported in other studies (Cox, Pyne, Gleson, \& Callister, 2008; Cullen, Thomas, Webb, \& Hughes, 2015; Minetto et al., 2005; Rossi, 2006). Therefore, it is reasonable to state that our training protocol was designed and executed correctly and our initial biomarker levels were appropriately normal, and hence are not confounding factors in this study's outcomes. This leaves the question why then did we see significant biomarker changes, which according to theory should reflect overtraining, but the overtrained state did not develop?

Evidence suggests the feasibility of the "Cytokine Hypothesis" as a causative means for developing overtraining is sound and logical (Robson, 2003; Robson-Ansley, Blannin, \& Gleeson, 2007; Robson-Ansley, de Milander, Collins, \& Noakes, 2004; Smith, 2000). We feel, however, that what is lacking is development of an understanding towards the magnitude of biomarker changes necessary to reflect the overtrained state. In other words, not just a direction of change, but how much of a change from normal healthy values is necessary to signal adaptive disruption. This type of recommendation is lacking in the literature and is vitally needed to allow for biomarker monitoring to become more viable as a means for use in the monitoring of athletes.

\section{CONCLUSIONS}

Current findings point to the need for investigators to recognize that the directional changes of endocrine-immune biomarkers may not reflect a pathology state until the magnitude of that change reaches a critical level. Therefore, criteria "cut-points" for the magnitude of such changes are needed and future research should address this point to improve the diagnostic capability of biomarkers in sports physiology.

Acknowledgements. This study received no external support.

Conflict of interest. The authors declare that they have no conflict of interest.

Ethical standards. The experiments conducted in this study comply with all guidelines and regulations within the USA.

\section{REFERENCES}

Burke, D. G., Chilibeck, P. D., Davidson, K. S., Candow, D. G., Farthing, J., \& Smith-Palmer, T. (2001). The effect of whey protein supplementation with and without creatine monohydrate combined with resistance training on lean tissue mass and muscle strength. International Journal of Sport Nutrition and Exercise Metabolism, 11(3), 349-364.

Cohen, J. (1992). A power primer. Psychological Bulletin, 112(1), 155-159.

Cox, A. J., Pyne, D. B., Gleson, M., \& Callister, R. (2008). Resting plasma and salivary IL-6 concentrations are not correlated in distance runners. European Journal of Applied Physiology, 103(4), 477-479. doi: 10.1007/ s00421-008-0722-X

Cullen, T., Thomas, A. W., Webb, R., \& Hughes, M. G. (2015). The relationship between interleukin-6 in saliva, venous and capillary plasma, at rest and in response to exercise. Cytokine, 71(2), 397-400. doi: 10.1016/j. cyto.2014.10.011

Fry, A. C., \& Kraemer, W. J. (1991). Physical performance characteristics of American collegiate football players.
The Journal of Strength \& Conditioning Research, 5(3), 126-138.

Golding, L. A. (Ed.). (2000). YMCA fitness testing and assessment manual (4 ${ }^{\text {th }}$ ed.). Champaign, IL: Human Kinetics.

Hackney, A. C. (2006). Stress and the neuroendocrine system: The role of exercise as a stressor and modifier of stress. Expert Review of Endocrinology and Metabolism, 1(6), 783-792. doi: 10.1586/17446651.1.6.783

Hackney, A. C., \& Viru, A. (2008). Research methodology: Endocrinologic measurements in exercise science and sports medicine. Journal of Athletic Training, 43(6), 631. doi: 10.4085/1062-6050-43.6.631

Hackney, A., \& Waltz, E. (2013). Hormonal adaptation and the stress of exercise training: The role of glucocorticoids. Trends in Sports Science, 4(20), 165171.

Hoffman, J. R., \& Kang, J. (2003). Strength changes during an in-season resistance-training program for football. Journal of Strength and Conditioning Research, 17(1), 109-114. 
Kraemer, W. J., \& Ratamess, N. A. (2005). Hormonal responses and adaptations to resistance exercise and training. Sports Medicine, 35(4), 339-361.

Lehmann, M., Foster, C., \& Keul, J. (1993). Overtraining in endurance athletes: A brief review. Medicine and Science in Sports and Exercise, 25(7), 854-862.

Meeusen, R., Duclos, M., Gleeson, M., Rietjens, G., Steinacker, J., \& Urhausen, A. (2006). Prevention, diagnosis and treatment of the Overtraining Syndrome. European Journal of Sport Science, 6(1), 1-14. doi:10.1080/17461390600617717

Minetto, M., Rainoldi, A., Gazzoni, M., Terzolo, M., Borrione, P., Termine, A., . . Paccotti, P. (2005). Differential responses of serum and salivary interleukin-6 to acute strenuous exercise. European Journal of Applied Physiology, 93(5-6), 679-686. doi: 10.1007/s00421-004-1241-z

Robson-Ansley, P. J., Blannin, A., \& Gleeson, M. (2007). Elevated plasma interleukin-6 levels in trained male triathletes following an acute period of intense interval training. European Journal Applied Physiology, 99(4), 353-360.

Robson-Ansley, P. J., de Milander, L., Collins, M., \& Noakes, T. D. (2004). Acute interleukin-6 administration impairs athletic performance in healthy, trained male runners. Canadian Journal of Applied Physiology, 29(4), 411-418.

Robson, P. (2003). Elucidating the unexplained underperformance syndrome in endurance athletes: The interleukin-6 hypothesis. Sports Medicine, 33(10), 771-781.

Rossi, S. J. (2006). Plasma and salivary cytokine response to two competitive collegiate soccer games. (Doctor of Philosophy Dissertation). Oklahoma State University.

Smith, L. L. (2000). Cytokine hypothesis of overtraining: A physiological adaptation to excessive stress? Medicine and Science in Sports and Exercise, 32(2), 317-331.

Urhausen, A., Gabriel, H., \& Kindermann, W. (1995). Blood hormones as markers of training stress and overtraining. Sports Medicine, 20(4), 251-276.

Ware, J. S., Clemens, C. T., Mayhew, J. L., \& Johnston, T. J. (1995). Muscular endurance repetitions to predict bench press and squat strength in college football players. Journal of Strength and Conditioning Research, 9(2), 99-103. 